\title{
Haptic unilateral and bilateral discrimination of curved surfaces
}

\author{
Astrid M L Kappers, Jan J Koenderink \\ Helmholtz Instituut, Princetonplein 5, 3584 CC Utrecht, The Netherlands; \\ e-mail: a.m.l.kappers@fys.ruu.nl \\ Received 6 September 1995, in revised form 29 February 1996
}

\begin{abstract}
Active haptic discrimination of cylindrically curved hand-sized surfaces was investigated. Unilateral discrimination (that is, with one hand, either the left or the right one) was compared with bilateral discrimination. In this latter condition, the right hand had to touch the right surface, and the left hand the left one. The importance of which surface of the pair is placed to the right of the observer and which to the left (the so-called placement order) was also investigated.

In comparison with existing studies, performance in the present experiments was better owing to the combined effects of a larger surface area and active instead of passive discrimination. The results show clearly that curvature discrimination does not follow a Weber law; performance is relatively better with the larger curvatures. For all subjects, unilateral discrimination was much better than bilateral discrimination. A partial cause of this difference is the influence of the placement order, which is very apparent in the results for the bilateral condition. These results cannot be fully explained but the findings suggest an influence of the object-observer relation on the perception of the object.
\end{abstract}

\section{Introduction}

Haptic manipulation and exploration of objects is an important aspect of dealing with our environment. However, compared with, for instance, vision and audition, touch has received relatively little attention (eg Loomis and Lederman 1986). Katz (1925), Révész (1938), Gibson (1962), and, more recently, Klatzky et al (1985) all realised the importance of active haptic exploration, but quantitative data on haptic perception hardly exist. In two recent studies (Kappers et al 1994a, 1994b), we investigated active haptic identification and discrimination of mathematically welldefined doubly curved surfaces. Here, we intend to pursue this line of research by investigating active discrimination performance with cylindrically curved surfaces.

The aim of this paper is to explore the following four issues: (1) how well are we able to actively discriminate cylindrically curved surfaces, (2) does curvature discrimination show Weber-like behaviour, (3) is there a difference between unilateral and bilateral discrimination, and (4) does the manner in which the surfaces are placed with respect to the observer influence performance?

With respect to the first issue, a number of studies are of relevance. Davidson (1972) describes experiments in which subjects had to categorise curved strips of $20 \mathrm{~cm}$ length as convex, straight, or concave. He focused on differences in performance between blind and sighted observers, and related that to differences in exploratory strategies. Unfortunately, it is not possible to derive from his data how much a strip has to be curved in order to be discriminable from a straight one. Davidson and Whitson (1974) investigated haptic matching of curvature for the same strips as in the previous study. Again, they do not give data on the dependence of performance on curvature, but show how performance depends on haptic strategy. Gordon and Morison (1982) tested active curvature-discrimination performance of the second finger of the preferred hand. The stimulus consisted of a curved strip of $10 \mathrm{~mm} \times 20 \mathrm{~mm}$. With results averaged over all subjects, they found that a surface 
with a curvature ${ }^{(1)}$ of $2 \mathrm{~m}^{-1}$ is discriminable from a flat surface. ${ }^{(2)}$. Also, a surface with a curvature of $2.7 \mathrm{~m}^{-1}$ could be distinguished from a surface with a curvature of $4.9 \mathrm{~m}^{-1}$. For passive curvature discrimination by the finger, Goodwin et al (1991) report that a flat surface could be distinguished from a convex surface with a curvature of $4.9 \mathrm{~m}^{-1}$, and from a concave one with curvature $-5.4 \mathrm{~m}^{-1}$. They also found that convex spherical surfaces with curvatures of $144 \mathrm{~m}^{-1}$ and $158 \mathrm{~m}^{-1}$ could be discriminated, just as surfaces with curvatures of $287 \mathrm{~m}^{-1}$ and $319 \mathrm{~m}^{-1}$. Thresholds of about the same magnitude were found when contact area remained constant (Goodwin and Wheat 1992). Last, Pont et al (1995) measured curvature discrimination of strips $20 \mathrm{~cm}$ long presented statically to different parts of the hand. Performance was best for strips placed along (as compared with perpendicular to) the fingers where a strip with a curvature of about $0.4 \mathrm{~m}^{-1}$ could be distinguished from a flat one. Although the total amount of data is still very sparse, it is tempting to draw some preliminary conclusions from these studies. If we compare the studies of Gordon and Morison (1982) and Goodwin et al (1991), it seems that active discrimination leads to better performance than passive discrimination. From a comparison of the results of Pont et al (1995) and, again, Goodwin et al (1991), it follows that discrimination of small surfaces is more difficult than that of larger ones.

The size of our stimuli is chosen such that they are roughly hand sized $(20 \mathrm{~cm}$ diameter). Since their areas are much larger than all the ones used in the abovementioned studies, we enter a new domain of surfaces. It is hard to predict whether or not performance will further improve.

Our second question, to investigate if curvature discrimination follows a Weber law, cannot be answered on the basis of previous investigations. Goodwin et al (1991) reported for their most curved stimuli (curvature between $149 \mathrm{~m}^{-1}$ and $319 \mathrm{~m}^{-1}$ ) a Weber fraction of 0.1 . Goodwin and Wheat (1992) give fractions of 0.13 for curvatures around $286 \mathrm{~m}^{-1}$, and 0.18 for curvatures around $154 \mathrm{~m}^{-1}$. Although they measured only around two standard curvatures, their data suggest that discrimination performance is relatively better (though only slightly so) for larger curvatures. We cannot simply extrapolate their findings to our experiments, since the curvatures of our stimuli lie necessarily in a totally different range (it is impossible to create stimuli of $20 \mathrm{~cm}$ diameter with curvatures over $10 \mathrm{~m}^{-1}$, since a curvature of $10 \mathrm{~m}^{-1}$ corresponds to a sphere with a diameter of $20 \mathrm{~cm}$ ).

The third issue deals with a possible difference between unilateral and bilateral discrimination. In unilateral discrimination (discrimination with one hand, either the left or the right one), surfaces can only be touched successively which suggests a disadvantage as compared with bilateral discrimination where the surfaces can be touched simultaneously. However, a previous study of discrimination of doubly curved surfaces clearly showed better performance for discrimination with only one hand (Kappers et al 1994b). A similar advantage of unilateral performance was found by Appelle and Countryman (1986) in a completely different experiment, namely the matching of the orientation of rods. Also, Fagot et al (1994) report that the accuracy in same-different judgments was greater if the objects were touched with only one hand. As a possible cause for this effect, Kappers et al (1994b) showed that it made a significant difference to performance if the locations of the two shapes which had to be discriminated were interchanged (in both cases, the right hand touched the right shape, and left hand the left one). This indicates that it matters which surface is touched by the right hand and which by the left. Our fourth aim is suggested by this finding: do we find a similar influence of location in curvature-discrimination experiments?

(1) Curvature is defined as reciprocal radius. That means that a sphere with a radius of $0.5 \mathrm{~m}$ has a curvature of $1 / 0.5 \mathrm{~m}^{-1}=2 \mathrm{~m}^{-1}$. Similarly, the curvature along the most curved direction of a cylinder with a radius of $0.5 \mathrm{~m}$ is also $2 \mathrm{~m}^{-1}$.

${ }^{(2)}$ They gave their results in terms of arc heights and diopters; the conversion to curvature is ours. 


\section{Methods}

\subsection{Stimuli}

The stimuli consisted of a set of cylindrically curved surfaces. The surfaces $z(x, y)$ can be described as $z(x, y)=-\frac{1}{2} \kappa x^{2}$ (no dependence on $y$ ) where $\kappa$ is the curvature. In order to define concave and convex, we use the convention that the material of the object occupies $z<z(x, y)$. In this manner, a negative (positive) curvature belongs to a concave (convex) surface. In anticipation of Weber-like behaviour, consecutive surfaces differed by a factor of $2^{1 / 2}$ in curvature, rather than having a constant spacing between them. In addition, we included a surface with zero curvature (a flat surface). For this experiment, surfaces with the following large range of curvatures were available: -5.7 , $-4,-2.8,-2,-1.4,-1,-0.7,-0.5,-0.35,0,0.35,0.5,0.7,1,1.4,2,2.8,4$, and $5.7 \mathrm{~m}^{-1}$.

The stimuli were made out of polyurethane foam impregnated with synthetic resin. The mechanical structure of this composite is similar to that of beech wood. The bottom of the stimuli was flat and always rested on the table; the top was the smooth curved surface. The diameter of all stimuli was $20 \mathrm{~cm}$. The surfaces were manufactured by means of a computer-controlled milling machine.

\subsection{Procedure}

Subjects were seated at a table behind a curtain so that both the experimenter and the curved surfaces were hidden from view. According to the condition, the subject put the right or left hand or both hands under the curtain for exploration of the surfaces. Stimuli, always presented in pairs to the subject, were placed next to each other in front of the subject.

The task of the subject was to determine which of the two simultaneously presented surfaces had the largest curvature. In order to avoid response confusions with the negatively curved surfaces (whether or not to take the absolute value of the curvature), the subject was given the following response alternatives: (a) right surface most convex, (b) left surface most convex, (c) right surface most concave, and (d) left surface most concave. Although responses (a) and (d), and also (b) and (c) are equivalent, subjects found it more convenient to use all four response possibilities. In practice, they used (a) and (b) for discrimination of convex surfaces, and (c) and (d) for the concave ones.

When both subject and experimenter were ready for a trial, the experimenter said "go" and at the same time pressed the button of a chronometer. The subject started exploring the surfaces until after $15 \mathrm{~s}$ the chronometer signalled the end of the exploration time by a short beep. The subject then noted down one of the alternatives on a special response sheet. Subjects were free to explore the surfaces in whatever way they preferred (using only their fingertips or their whole hand etc.), provided that the contours of the surface were not scanned systematically. ${ }^{(3)}$ This latter requirement was only necessary in order to avoid the strategy of direct height comparisons at the contours; given the nature of our stimuli, this should not be considered as a serious constraint. The flat bottom of each shape remained on the table. In practice, most subjects tried to align the cylindrical surfaces either along or perpendicular to the direction of their fingers by rotating the surfaces.

Three different conditions were tested. In condition 1, the subjects were only allowed to use their left hand, in condition 2 they had to use their right hand, and in condition 3 they had to use both hands. In the last condition, the right hand touched the right surface and the left hand the left one. Subjects were not allowed to cross their hands (left hand touching the right surface and vice versa). In condition 3 , it was left up to the subjects whether they explored the surfaces simultaneously or successively, but total exploration time remained fixed at $15 \mathrm{~s}$.

(3) In practice, all subjects palpated the surface with the entire hand instead of using only their fingers. Although free to do otherwise, subjects always made scanning movements. No attempt was ever made to base responses on information provided by a static hand. 
The pairs of stimuli presented to the subjects always had successive curvatures from the range given above. Since there are nineteen different surfaces, there are eighteen different pairs. Each pair was presented sixteen times to the subject, eight times with the more convex surface of the two on the right side of the subject, and eight times on the left side. All pairs were presented in random order. Each condition consisted of four sessions of about an hour. The sessions took place on different days.

\subsection{Subjects}

Four subjects participated in our experiments. The first author was one of the subjects; the other three were physics majors who participated on a voluntary basis. According to the definition used by Coren (1993), they were all strongly right-handed. None of the subjects reported any haptic deficiencies.

\section{Results}

In figure 1 , we present the results of one subject. For reasons which will soon become obvious, results of the other subjects will be presented later in this paper in a slightly modified way. Figure 1 shows the percentages of correct judgments of curvature discrimination for all the different curvature pairs. It can clearly be seen that overall performance is quite good. For many curvature pairs $100 \%$ correct judgments occur.
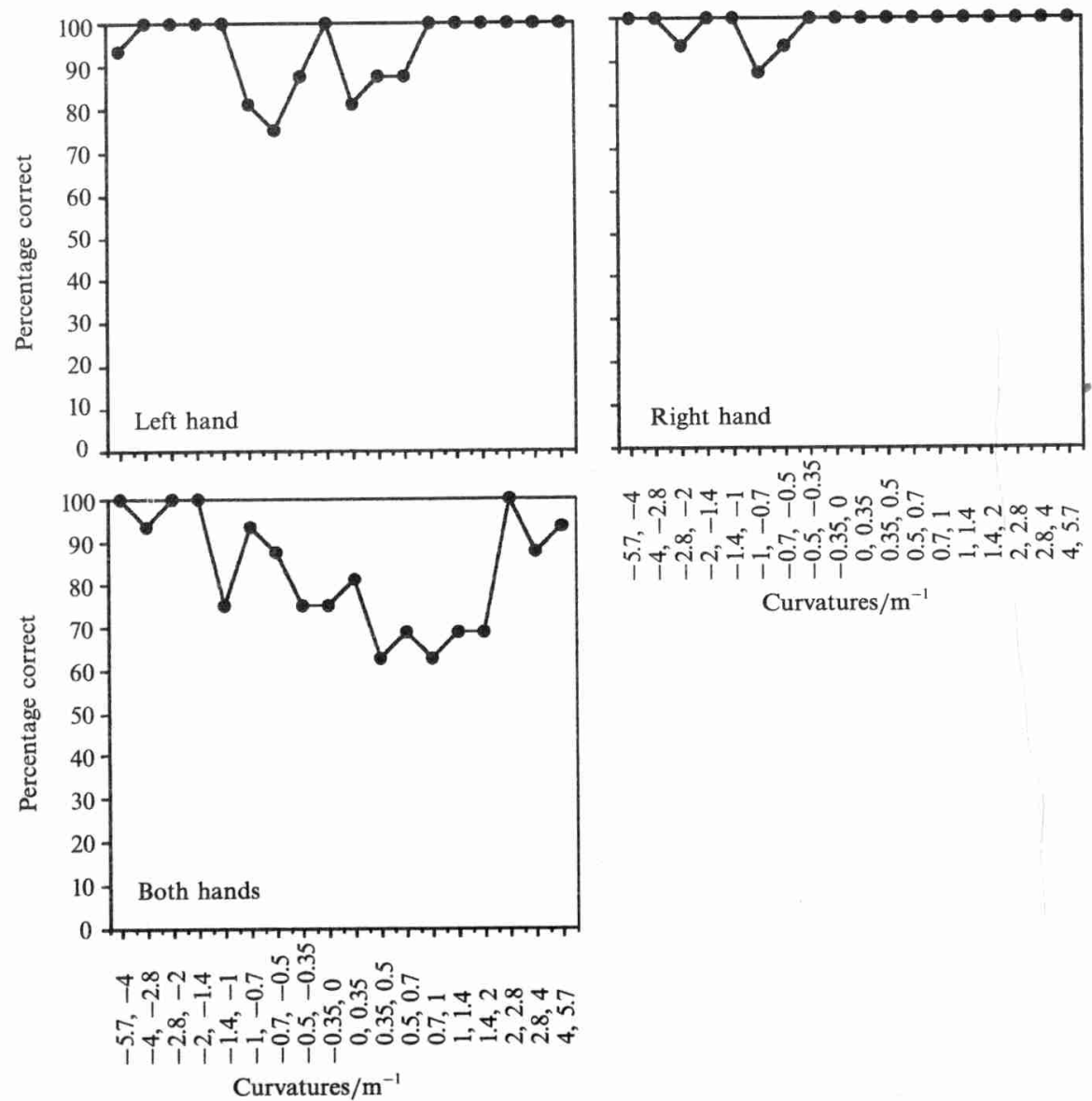

Figure 1. Percentages of correct curvature-discrimination judgments of subject AK for all curvature pairs, and for discrimination with the left hand, with the right hand, and with both hands. 
Percentages of correct judgments as low as $65 \%$ only occur in the condition where both hands had to be used. Apparently, performance for unilateral discrimination is better than for bilateral discrimination. In order to facilitate comparisons between performances in the three different conditions, we calculated for each subject the percentages of correct judgments averaged over all curvature pairs. The results are shown in figure 2 where each curve corresponds to a particular condition. This figure shows convincingly that for all subjects performance is worse if two hands have to be used in this discrimination task. There is no such difference between performances of the two unilateral conditions.

Since neighbouring curvatures differ by a constant factor (except around zero curvature), we expect a constant level of performance if a Weber law holds for curvature discrimination. From the results presented in figure 1 it is difficult to draw a straightforward conclusion, but this figure certainly suggests that performance is best for pairs with the largest curvatures. We have quantified this difference by calculating separately the average percentage of correct judgments for pairs with small, medium, and large curvatures. Since we only aim at getting a rough indication of whether or not there is an effect of curvature, the actual division between large, medium, and small curvatures is not important. We have chosen absolute curvatures of $0.7 \mathrm{~m}^{-1}$ and $2 \mathrm{~m}^{-1}$ as the boundaries between small and medium, and medium and large, respectively, so that six pairs fall in each category. Thus, the 'small' category consists of all pairs with curvatures between $-0.7 \mathrm{~m}^{-1}$ and $+0.7 \mathrm{~m}^{-1}$; the 'medium' category consists of all pairs with curvatures between $-2 \mathrm{~m}^{-1}$ and $-0.7 \mathrm{~m}^{-1}$, and between $+0.7 \mathrm{~m}^{-1}$ and $+2 \mathrm{~m}^{-1}$, and the 'large' category contains all pairs with curvature between $-5.7 \mathrm{~m}^{-1}$ and $-2 \mathrm{~m}^{-1}$, and between $+2 \mathrm{~m}^{-1}$ and $+5.7 \mathrm{~m}^{-1}$. The resulting percentages are shown in table 1 . Once again, the results are very convincing: for all subjects and under all conditions, the percentages of correct judgments for the pairs with the smaller curvatures lie below those of the pairs with the medium curvatures, and similarly the latter ones lie below the scores reached with the largest curvature (the only exception is the score for the medium category of AK using her right hand). Unfortunately, our results do not allow a more detailed analysis of the dependence of performance on curvature.

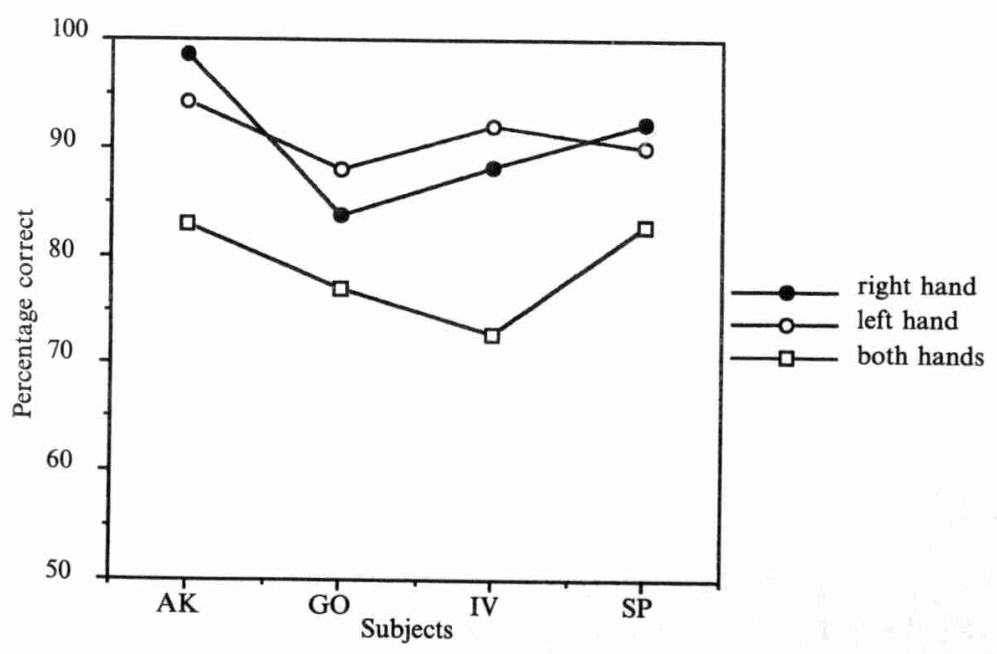
Figure 2. Average percentage of correct curvature-discrimination judgments of all four subjects
for all conditions. 
Table 1. Average percentages of correct curvature-discrimination judgments for the four subjects in all conditions. A distinction is made between scores for pairs with large, medium, and small absolute curvature. The small-curvature pairs consist of the six pairs with curvatures between $-0.7 \mathrm{~m}^{-1}$ and $+0.7 \mathrm{~m}^{-1}$. The six medium-curvature pairs have curvatures between $-2 \mathrm{~m}^{-1}$ and $-0.7 \mathrm{~m}^{-1}$, or between $+0.7 \mathrm{~m}^{-1}$ and $+2 \mathrm{~m}^{-1}$. The large-curvature pairs are the six remaining pairs, with curvatures between $-5.7 \mathrm{~m}^{-1}$ and $-2 \mathrm{~m}^{-1}$ or between $+2 \mathrm{~m}^{-1}$ and $+5.7 \mathrm{~m}^{-1}$.

\begin{tabular}{|c|c|c|c|c|c|c|c|c|c|}
\hline \multirow[t]{2}{*}{ Subject } & \multicolumn{3}{|c|}{ Left hand } & \multicolumn{3}{|c|}{ Right hand } & \multicolumn{3}{|c|}{ Both hands } \\
\hline & small & medium & large & small & medium & large & small & medium & large \\
\hline $\mathrm{AK}$ & 86.5 & 96.9 & 99.0 & 99.0 & 97.9 & 99.0 & 75.0 & 78.1 & 95.8 \\
\hline GO & 75.0 & 89.6 & 99.0 & 65.6 & 89.6 & 95.8 & 69.2 & 76.0 & 85.4 \\
\hline IV & 82.3 & 95.8 & 97.9 & 82.3 & 86.5 & 95.8 & 60.4 & 74.0 & 83.3 \\
\hline SP & 78.1 & 93.8 & 97.9 & 83.3 & 94.8 & 97.9 & 63.5 & 86.9 & 97.9 \\
\hline Average & 80.5 & 94.1 & 98.5 & 82.6 & 92.2 & 97.1 & 67.0 & 78.8 & 90.6 \\
\hline
\end{tabular}
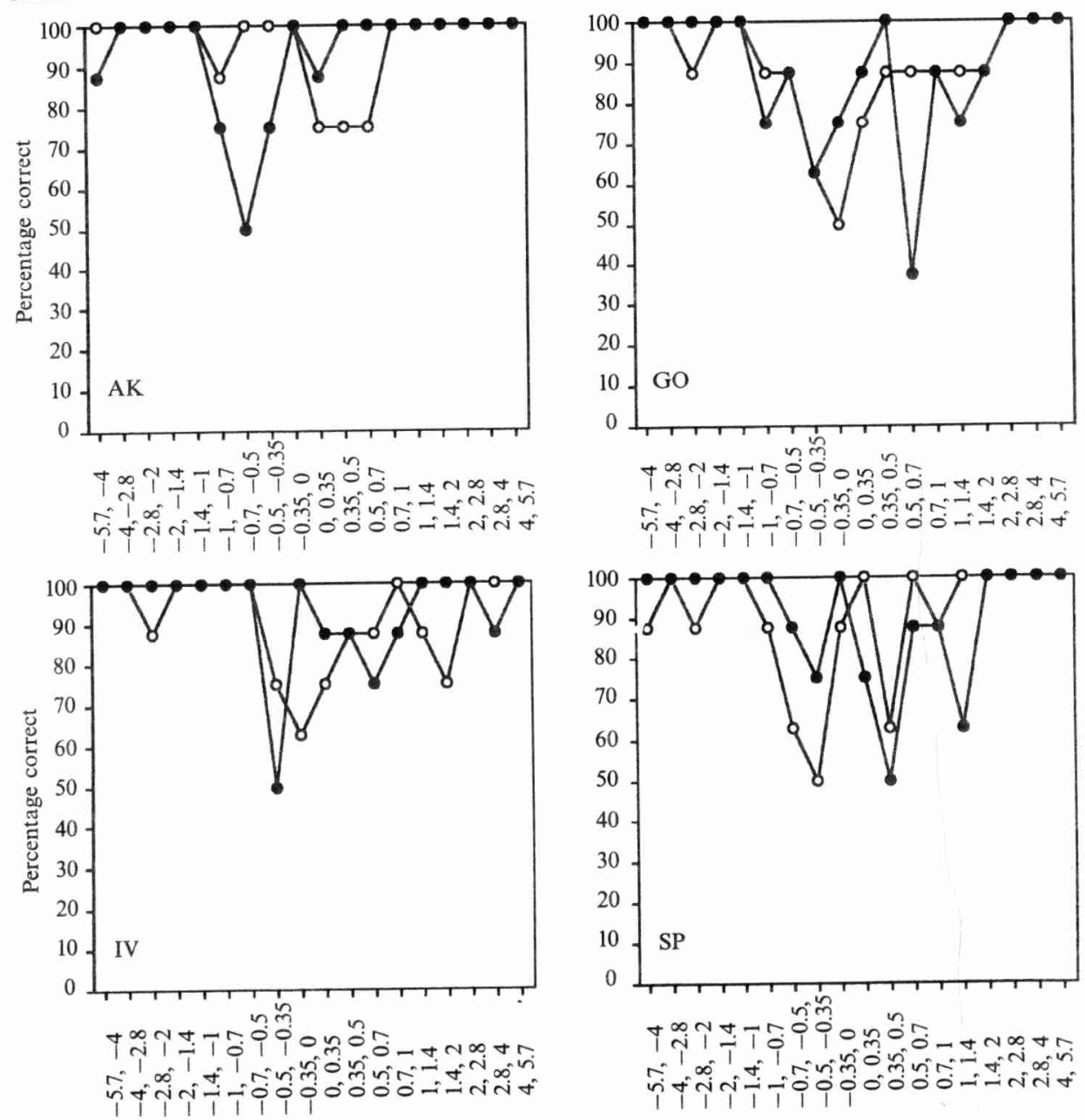

Curvatures $/ \mathrm{m}^{-1}$

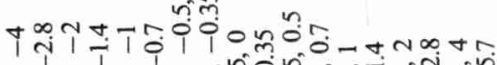

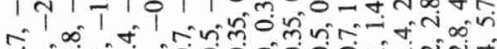

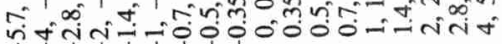

Curvatures $/ \mathrm{m}^{-1}$

Figure 3. Percentages of correct curvature discrimination judgments of all subjects using their left hand. The filled circles give the results for curvature pairs of which the right shape with respect to the subject had the largest curvature (that is, the right shape was the more convex of the two). The open circles give results for the opposite order of placement of the shapes with respect to the subject. 
We also investigated performance for convex and concave curvatures separately. Averaged over all subjects and all curvatures, performance with the left hand is $91 \%$ correct both for convex and for concave curvatures. For the right hand, percentages of correct judgments are $92 \%$ for concave and $90 \%$ for convex curvatures. So, apparently, in the unilateral conditions the sign of the curvature has no influence on performance. This is different, however, for the bilateral condition, where the scores are $84 \%$ and $74 \%$ correct for concave and convex, respectively. Such a difference was present in the results of all individual subjects, although it was not always $10 \%$. Thus, in the bilateral condition concave curvatures are somewhat better discriminable than convex ones.

As we showed in figure 2 and in table 1, unilateral performance is much better than bilateral performance. Following the suggestion from previous research that placement of the surfaces with respect to the subject could be of influence, we analysed the data
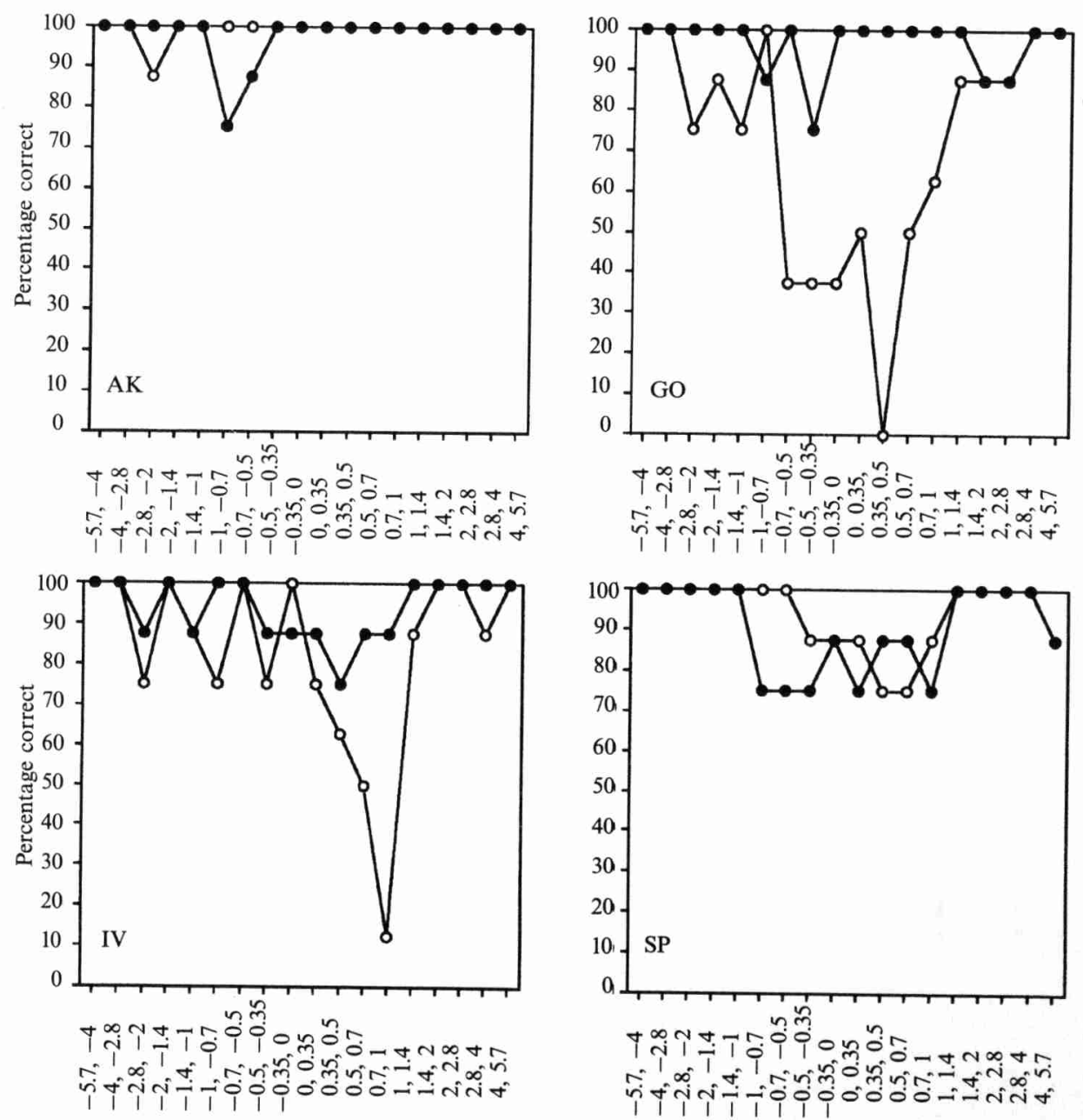

†

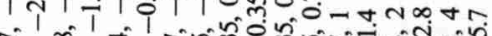

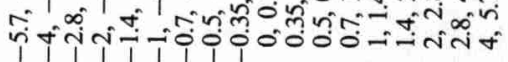

Curvatures $/ \mathrm{m}^{-1}$

Curvatures $/ \mathrm{m}^{-1}$

Figure 4. Percentages of correct curvature discrimination judgments of all subjects using their right hand. The filled circles give the results for curvature pairs of which the right shape with respect to the subject had the larger curvature (that is, the right shape was the more convex of the two). The open circles give results for the opposite order of placement of the shapes with respect to the subject. 
as follows. For each pair of curvatures we determined separately the percentage of correct judgments for the pairs in which the right surface had the larger curvature (was more convex) and for the opposite pairs (left surface more convex). The results are shown in figures 3, 4, and 5, for the left hand, the right hand, and both hands, respectively. The four graphs in each figure give the results for the four subjects. For the filled circles the more-convex surface was the right one, and for the open circles the more-convex surface was the left one. (Note that the average of the two curves results in curves like in figure 1.)

In figure 3 it can be seen that the placement of the surfaces with respect to the subject is not of influence if the discrimination task is performed with the left hand. For the right hand, however, the placement matters for two of the subjects (GO and IV), as can be seen in figure 4. Especially for GO, the task seems to be much easier if the more convex surface of the pair is placed to the right. Surprisingly, performance is sometimes even below chance $(50 \%$ correct) if the two surfaces are placed in the
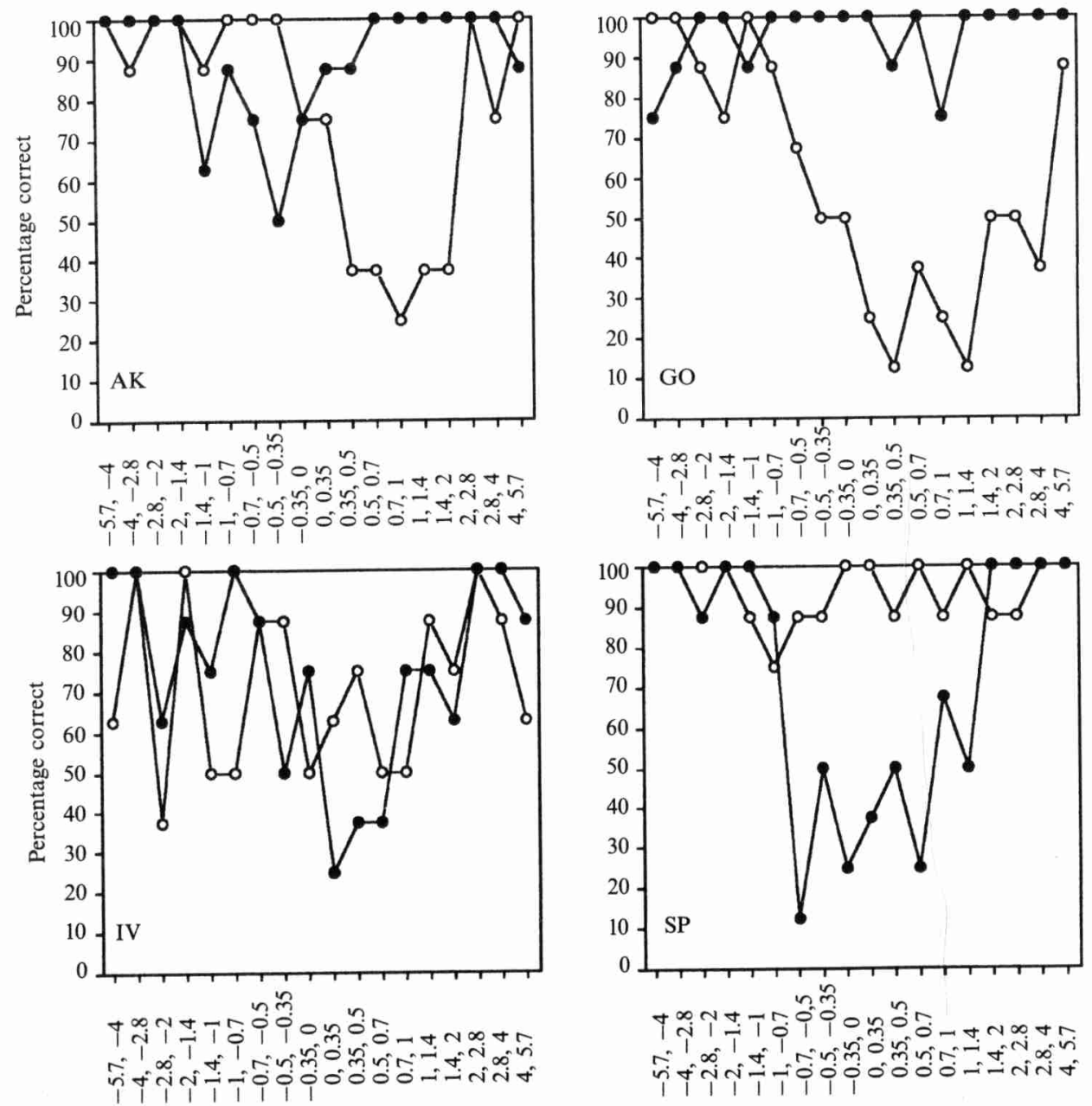

Curvatures $/ \mathrm{m}^{-1}$

Curvatures $/ \mathrm{m}^{-1}$

Figure 5. Percentages of correct curvature-discrimination judgments of all subjects using both hands. The filled circles give the results for curvature pairs of which the right shape with respect to the subject had the larger curvature (that is, the right shape was the more convex of the two). The open circles give results for the opposite order of placement of the shapes with respect to the subject. 
opposite order. The effect for subject IV is relatively small, since it is restricted to three curvature pairs (between $+0.35 \mathrm{~m}^{-1}$ and $+1 \mathrm{~m}^{-1}$ ). For discrimination with two hands, the order of placement is of strong influence for three of the four subjects, as is shown in figure 5. Again, performance is often close to $100 \%$ in one order, and below chance for the opposite order. Which of the two orders of placement results in best performance depends on the subject.

In order to quantify the placement-order effect, we computed biases for all conditions and all subjects. The bias is defined as the percentage of correct judgments averaged over all curvature pairs in which the right surface was more convex minus that for pairs where the left surface was more convex. The biases are shown in figure 6. What we already observed in figures 3,4 , and 5 becomes more apparent here. For two subjects (AK and SP) there is no bias for the left and the right hand, but there certainly is a clear bias for discrimination, with both hands. For these two subjects, the bias appears in opposite direction. For subject GO there is no bias for the left hand, a distinct bias for the right hand, and an even-stronger bias for two hands. Only for subject IV is there no bias for the two-hands condition, although there is a bias for the right-hand condition.

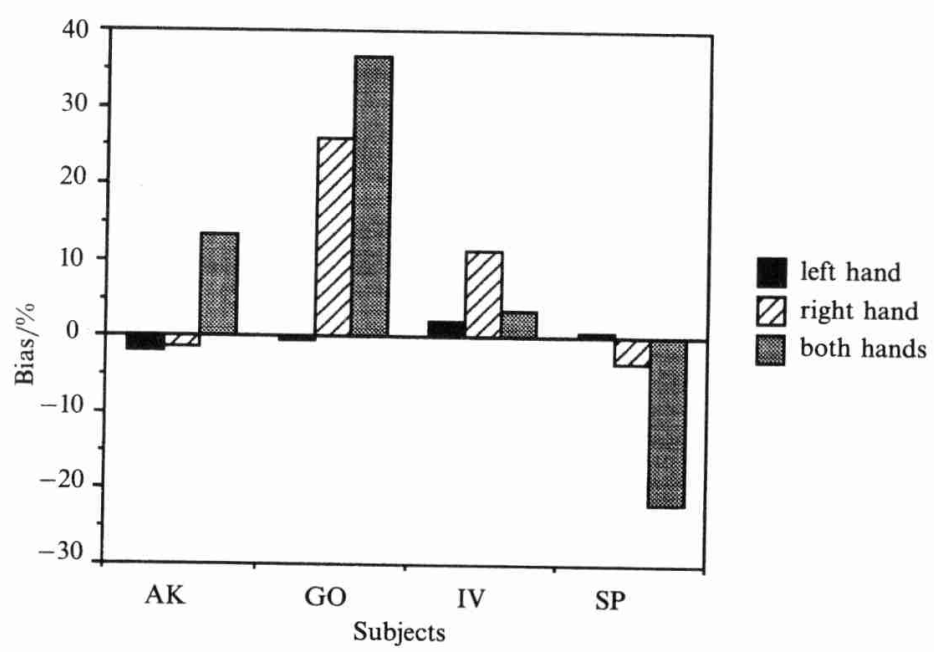

Figure 6. Biases of all four subjects for the three conditions. The bias is defined as the average percentage of correct discrimination judgments for curvature pairs of which the right shape has
the larger curvature minus that for opposite pairs.

\section{Discussion}

From our experiments we have got a good impression of human curvaturediscrimination abilities for hand-sized objects with curvatures common in daily life. The curvatures, which differed by a factor $2^{1 / 2}$ (except, of course, around 0 ), could be discriminated in $90 \%$ of the trials when the task was performed with either the left or the right hand, and $79 \%$ of the trials when discriminating with both hands (averaged over all subjects and curvatures). This is much better than the performance reported by Gordon and Morison (1982) for active discrimination of small curved surfaces with just one finger $\left(0 \mathrm{~m}^{-1}\right.$ can be discriminated from $2 \mathrm{~m}^{-1}$, and $0.4 \mathrm{~m}^{-1}$ from $\left.2.7 \mathrm{~m}^{-1}\right)$. For discrimination of very large curvatures applied to the passive finger, Goodwin et al (1991) and Goodwin and Wheat (1992) reported that for $75 \%$ correct performance, curvature differences of a factor of about 1.13 are sufficient. A direct comparison with our results is difficult, since our curvatures differ by a factor $1.41\left(=2^{1 / 2}\right)$ and performance is better than $75 \%$. Still, the results suggest that performance in the experiments 
of Goodwin et al and Goodwin and Wheat is better than in ours. However, one should keep in mind that their stimuli really lie in a different range; a stimulus with a curvature of, for instance, $286 \mathrm{~m}^{-1}$ has a radius of $3.5 \mathrm{~mm}$ which is not at all handsized! In comparing our results with those of Pont et al (1995), it seems that active discrimination of hand-sized objects leads to slightly better performance than passive discrimination of curved strips. Summarising, we can say that within the range of curvatures used in our experiments, performance increases with size. Furthermore, active discrimination leads to better performance than passive discrimination.

In table 1 it can be seen that percentages of correct curvature-discrimination judgments depended on the curvature of the stimuli. Given the constant factor between the various curvatures, performance was relatively better with the larger curvatures. Goodwin and Wheat (1992) reported a similar finding for much larger curvatures. Moreover, performance in their experiment seems to be relatively better than in ours. Taken together, this provides ample evidence that curvature discrimination does not follow a Weber law.

From both figure 2 and table 1 it can be seen convincingly that for all subjects bilateral performance is much worse than unilateral performance. In contrast, performances with the left and the right hand separately are quite comparable (ceiling effects might obscure some minor differences between the two hands, but that is not of any relevance for the present study). Apparently, the supposed advantage of simultaneous discrimination does not compensate for the extra difficulty introduced by the need to compare information gained with different hands. This finding is in agreement with one of our previous studies (Kappers et al 1994b), and also with results of, for instance, Appelle and Countryman (1986) and Fagot et al (1994). Appelle and Countryman concluded that in their experiment the difference between unilateral and bilateral performance is caused by the inherently different scanning patterns required in the two conditions. Since our surfaces can be rotated to the subject's preferred orientation, we do not think that different scanning patterns can explain the differences we found. In the experiments of Fagot et al, subjects had to compare three-dimensional configurations of cubes and decide whether they were the same or different. They found that, in their setup, subjects touched regions ipsilateral to the hand more often than contralateral regions. As a consequence, in bilateral discrimination different regions of the object were compared which had a deteriorating effect on performance. The fact that our surfaces are symmetrical excludes such an explanation for our results.

Previous research (Kappers et al 1994b) suggested that in bilateral experiments it might be of influence which surface is touched with the left hand and which with the right. It can also be seen from figure 5 that in curvature-discrimination experiments also this is indeed an important factor. For three of the four subjects, discrimination performance is close to $100 \%$ in one placement order, and often even below chance in the opposite order. A possible explanation could be that it simply shows a response bias. However, this seems unlikely, as it cannot explain the results obtained in our shapediscrimination experiments (Kappers et al 1994b). There the question was whether two shapes were the same or different, and we only analysed all different pairs for a placement-order effect. Moreover, in the experiments described here, one would expect similar biases in the unilateral conditions. Thus, although we cannot fully exclude a response bias, we deem it necessary to search for alternative explanations. Consider a very crude model for the discrimination of curvatures: quantities which have some monotonic relationship with curvature are compared (at which level this comparison actually takes place is not important). If, in such a model, the channels subserving the two hands have different gain factors, this would favour one placement order with respect to the other, without affecting unilateral performance. Moreover, such a cause could influence performance to different degrees and in different directions for different subjects. 
Prerequisite for such an explanation is that the placement order is not of any influence in the unilateral conditions. For two subjects this is indeed the case, but two others show placement-order effects for their right hand, although for one of them the effect is relatively small. Apparently, an explanation in terms of such a model cannot be the whole story. What remains is the unavoidable conclusion that the position of an object with respect to the observer can be of influence in a curvature-discrimination task. Touching a surface to the right in front or to the left in front of you might make a difference in estimating the curvature.

Except for the study of Goodnow et al (1971) where the exploratory movements were severely constrained, we do not know of any study in which haptic perception was investigated as a function of the position of the object with respect to the body. An experiment which might be of relevance here is reported by Soechting et al (1986). They showed that the circles drawn by subjects on different planes with respect to their bodies are distorted in a characteristic way. Motor coordination played a major role in their explanations. Investigating in more detail the influence on perception of the object - observer relation seems to be a challenging direction for future research.

Acknowledgement. This research is supported by the Netherlands Organization for Scientific Research (NWO). The authors wish to thank Gerlene Oudenaarden, Susan F te Pas and Ingrid M L C Vogels for their valuable contribution.

\section{References}

Appelle S, Countryman M, 1986 "Eliminating the haptic oblique effect: influence of scanning incongruity and prior knowledge of the standards" Perception 15 325-329

Coren S, 1993 The Left-hander Syndrome (New York: Vintage Books)

Davidson P W, 1972 "Haptic judgments of curvature by blind and sighted humans" Journal of Experimental Psychology $9343-55$

Davidson P W, Whitson T T, 1974 "Haptic equivalence matching of curvature by blind and sighted humans" Journal of Experimental Psychology $102687-690$

Fagot J, Lacreuse A, Vauclair J, 1994 "Hand-movement profiles in a tactual-tactual matching task: Effects of spatial factors and laterality" Perception \& Psychophysics 56 347-355

Goodnow J J, Baum B, Davidson P, 1971 "A haptic error: Skew in a symmetrical curve" Perception \& Psychophysics $10253-256$

Goodwin A W, John K T, Marceglia A H, 1991 "Tactile discrimination of curvature by humans using only cutaneous information from the fingerpads" Experimental Brain Research 86 $663-672$

Goodwin A W, Wheat H E, 1992 "Human tactile discrimination of curvature when contact area with the skin remains constant" Experimental Brain Research 88 447-450

Gordon I A, Morison V, 1982 "The haptic perception of curvature" Perception \& Psychophysics $31446-450$

Gibson J J, 1962 “Observations on active touch" Psychological Reviews 69 477-491

Kappers A M L, Koenderink J J, Lichtenegger I, 1994a "Haptic identification of curved surfaces" Perception \& Psychophysics 56 53-61

Kappers A M L, Koenderink J J, te Pas S F, 1994b "Haptic discrimination of doubly curved surfaces" Perception 231483 - 1490

Katz D, 1925 Der Aufbau der Tastwelt (Leipzig: Barth)

Klatzky R L, Lederman S J, Metzger V A, 1985 "Identifying objects by touch: An "expert system" "Perception \& Psychophysics $37299-302$

Loomis J M, Lederman S J, 1986 "Tactual perception", in Handbook of Perception and Human Performance Volume II Cognitive Processes and Performance Eds K R Boff, L Kaufman, J P Thomas (New York: John Wiley)

Pont S C, Kappers A M L, Koenderink J J, 1995 "Haptic discrimination of curved strips", in Studies in Perception and Action III Eds B G Bardy, R J Bootsma, Y Guiard (Hillsdale, NJ: Lawrence Erlbaum Associates) pp 307-310

Révész G, 1938 Die Formenwelt des Tastsinnes (Dordrecht: Martinus Nijhoff)

Soechting J F, Lacquaniti F, Terzuolo C A, 1986 "Coordination of arm movements in threedimensional space. Sensorimotor mapping during drawing movement" Neuroscience 17 $295-311$ 